\title{
Proteome-Wide Analyses Reveal the Diverse Functions of Lysine 2- Hydroxyisobutyrylation in Oryza sativa
}

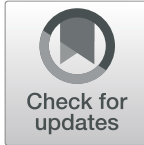

Chao Xue ${ }^{1,2+}$, Zhongying Qiao ${ }^{3 \dagger}$, Xu Chen ${ }^{1,2}$, Penghui Cao ${ }^{3}$, Kai Liu ${ }^{1,2}$, Shuai Liu ${ }^{1,2}$, Lu Ye $^{1,2}$ and Zhiyun Gong ${ }^{1,2^{*}}$ (D)

\begin{abstract}
Background: Lysine 2-hydroxyisobutyrylation (Khib), a newly identified post-translational modification, is known to regulate transcriptional activity in animals. However, extensive studies of the lysine 2-hydroxyisobutyrylome in plants and animals have yet to be performed.

Results: In this study, using LC-MS/MS qualitative proteomics strategies, we identified 4163 Khib sites on 1596 modified proteins in rice (Oryza sativa) seedlings. Motif analysis revealed 10 conserved motifs flanking the Khib sites, and subcellular localization analysis revealed that $44 \%$ of the Khib proteins are localized in the chloroplast. Gene ontology function, KEGG pathway, and protein domain enrichment analyses revealed that Khib occurs on proteins involved in diverse biological processes and is especially enriched in carbon metabolism and photosynthesis. Among the modified proteins, 20 Khib sites were identified in histone $\mathrm{H} 2 \mathrm{~A}$ and $\mathrm{H} 2 \mathrm{~B}$, while only one site was identified in histone H4. Protein-protein interaction (PPI) network analysis further demonstrated that Khib participates in diverse biological processes including ribosomal activity, biosynthesis of secondary metabolites, and metabolic pathways. In addition, a comparison of lysine 2-hydroxyisobutyrylation, acetylation, and crotonylation in the rice proteome showed that 45 proteins with only 26 common lysine sites are commonly modified by three PTMs. The crosstalk of modified sites and PPI among these PTMs may form a complex network with both similar and different regulatory mechanisms.
\end{abstract}

Conclusions: In summary, our study comprehensively profiles the lysine 2-hydroxyisobutyrylome in rice and provides a better understanding of its biological functions in plants.

Keywords: Oryza sativa, Lysine 2-hydroxyisobutyrylation, Photosynthesis, Histone acylation

\section{Background}

Posttranslational modifications (PTMs) of proteins play important roles in metabolic regulation, protein interaction, cell signaling, and other biological processes (Choudhary et al. 2014; Martin and Zhang 2005). In recent years, increasingly novel types of PTM on lysine residues have been

\footnotetext{
* Correspondence: zygong@yzu.edu.cn

${ }^{\dagger}$ Chao Xue and Zhongying Qiao contributed equally to this work.

'Jiangsu Key Laboratory of Crop Genetics and Physiology/ Key Laboratory of

Plant Functional Genomics of the Ministry of Education/ Jiangsu Key

Laboratory of Crop Genomics and Molecular Breeding, Agricultural College

of Yangzhou University, Yangzhou 225009, China

${ }^{2}$ Jiangsu Co-Innovation Center for Modern Production Technology of Grain

Crops, Yangzhou University, Yangzhou 225009, China

Full list of author information is available at the end of the article
}

identified and demonstrated to be associated with regulatory functions. These PTMs include lysine succinylation (Ksucc), butyrylation (Kbu), propionylation (Kpr), crotonylation (Kcr), malonylation (Kma), $\beta$-hydroxybutyrylation (Kbhb), and glutarylation (Kglu) (Chen et al. 2007; Tan et al. 2014; Xie et al. 2012; Xie et al. 2016; Zhang et al. 2011; Tan et al. 2011). Like acetylation (Kac), these emerging PTMs are classified as lysine acylations and have been demonstrated to occur on multiple proteins involved in various cellular metabolic processes (Lin et al. 2012; Weinert et al. 2013). A growing body of evidence indicates that lysine acylation or deacylation can be catalyzed by certain lysine acetyltransferases (KATs) and deacetylases (KDACs) (Huang et al. 2018a). Previous studies have revealed that

\section{Springer Open}

(๑) The Author(s). 2020 Open Access This article is licensed under a Creative Commons Attribution 4.0 International License, which permits use, sharing, adaptation, distribution and reproduction in any medium or format, as long as you give appropriate credit to the original author(s) and the source, provide a link to the Creative Commons licence, and indicate if changes were made. The images or other third party material in this article are included in the article's Creative Commons licence, unless indicated otherwise in a credit line to the material. If material is not included in the article's Creative Commons licence and your intended use is not permitted by statutory regulation or exceeds the permitted use, you will need to obtain permission directly from the copyright holder. To view a copy of this licence, visit http://creativecommons.org/licenses/by/4.0/. 
p300, a lysine acetyltransferase, can catalyze other acylations besides lysine acetylation, including crotonylation, butyrylation, propionylation, and 2-hydroxyisobutyrylation (Huang et al. 2018b; Sabari et al. 2018; Chen et al. 2007). Furthermore, many histone acylations have been shown to exhibit functions in modulating chromatin status and stimulating transcriptional activity (Sabari et al. 2018; Tan et al. 2011).

Lysine 2-hydroxyisobutyrylation (Khib) is a novel PTM first identified in human and mouse proteins as a widely distributed active histone mark (Dai et al. 2014). In this pioneering study, histone Khib was shown to have a different genomic distribution from those of histone Kac or Kcr during male germ cell differentiation. The structural differences of Khib from lysine methylation, acetylation, and crotonylation enable it to induce a larger change in modified-lysine size, and the hydroxyl group of Khib allows the modified lysine to form hydrogen bonds with other molecules, which is important for regulating protein functions (Dai et al. 2014; Maxwell et al. 1999). H4K8hib is associated with active gene transcription in mouse meiotic and post-meiotic cells. Furthermore, H4K8hib has been demonstrated to regulate gene transcriptional activity in Saccharomyces cerevisiae and is differentially regulated under different glucose concentrations (Huang et al. 2017). Besides, Khib in non-histones has been also involved in diverse biological processes, including the tricarboxylic acid cycle, glycolysis/gluconeogenesis, and especially enriched in mitochondrial proteins within energy metabolic networks (Huang et al. 2018a; Wu et al. 2018; Huang et al. 2018b). These findings indicate that Khib has regulatory functions on cellular processes and gene transcriptional activity. However, the regulatory mechanisms of this new PTM in diverse biological processes are not well understood for both eukaryotes and prokaryotes.

In recent decades, the application of high-specificity pan-antibodies and high-sensitivity mass spectrometry (MS) techniques have broadened the current catalogue of PTMs on histones and non-histones. MS analysis relies on the identification of modification-induced changes in the masses of peptides, which are termed mass shifts (Sabari et al. 2017). Once a mass shift caused by a PTM is detected or localized to certain residues, the chemical structure of the modifier can be deduced and validated through high performance liquid chromatography (HPLC) coelution, tandem mass spectrometry analysis, isotopic labeling, and immunochemistry using an appropriate antibody (Chick et al. 2015; Dai et al. 2014; Tsur et al. 2005). Accordingly, MS-based approaches have become highly efficient and powerful methods for large-scale analysis of PTMs and identifying novel lysine modifications.

For example, the first global profiling of the Khib proteome in mammalian cells identified 6548 Khib sites on 1725 substrate proteins (Huang et al. 2018a). By combining affinity enrichment with HPLC-MS, a total of 4735 lysine sites on 1051 proteins in Proteus mirabilis were found to be 2hydroxyisobutyrated, indicating that Khib may play important roles in bacterial metabolism (Dong et al. 2018). Furthermore, proteome-wide analysis of Khib in Physcomitrella patens identified 11,976 Khib sites in 3001 proteins involved in a wide range of molecular functions and cellular processes (Yu et al. 2017). Together, these findings show that Khib may have diverse and novel functions on biological processes in eukaryotes and prokaryotes.

Rice (Oryza sativa) is an important model organism for cereal research and provides food for more than half of the world's population (Ashikari et al. 2005). In recent years, rapid development of proteomics techniques and specific pan-antibodies have dramatically expanded the global profiling of the PTM proteome in rice, including the acetylome, succinylome, ubiquitome, crotonylome and 2hydroxyisobutyrylome (He et al. 2016; Liu et al. 2018; Meng et al. 2017; Xie et al. 2015; Xiong et al. 2016; Xue et al. 2018).

Our previous study identified 1353 Kac sites on 866 proteins in rice seedlings and demonstrated that H3 (lysine 27 and 36) acetylation increased in response to cold stress (Xue et al. 2018). Furthermore, the first proteomic analysis of lysine crotonylation in rice identified $1265 \mathrm{Kcr}$ sites on 690 proteins and proved that histone $\mathrm{Kcr}$ is positively correlated with gene expression in plants (Liu et al. 2018). In another study, a total of 699 acetylated sites on 389 proteins and 665 succinylated sites on 261 proteins were identified in the embryo of germinating rice seed using HPLC-MS/MS (He et al. 2016). More recently, a huge lysine 2-hydroxyisobutyrylome dataset with 9916 Khib sites on 2512 proteins was identified in developing rice seeds (Meng et al. 2017), providing the first systematic analysis of the Khib proteome and its potential regulatory functions for grain filling and development in rice. Like other acylations, Khib may have similar potential physiological functions on plant growth and development. However, the number of reports on Khib in plants is still very limited.

In this study, utilizing 2-hydroxyisobutyrylation affinity enrichment and LC-MS/MS qualitative proteomics strategies, we identified 4163 2-hydroxyisobutyryl lysine sites on 1596 proteins in rice seedlings. A detailed proteomic analysis revealed that Khib occurs in proteins involved in biological functions and metabolic processes. Through the crosstalk of the 2-hydroxyisobutyrylome, crotonylome, and acetylome in rice leaves, we found that these acylations have overlapping modified proteins and sites to participate in regulatory metabolisms, but also have significant distinctions to execute diverse protein functions.

\section{Results}

Proteome-Wide Analyses Revealed a Large Lysine 2Hydroxyisobutyrylome in Rice

Khib has been proved to be involved in diverse biological processes in eukaryotes and prokaryotes (Yu et al. 2017; 
Tan et al. 2011; Dai et al. 2014; Dong et al. 2018). To investigate the distribution of Khib modification in the proteins of rice, we performed Western blot assaying using pan - 2-hydroxyisobutyryllysine antibody (Fig. 1a). The sodium dodecyl sulfate polyacrylamide gel electrophoresis (SDS-PAGE) and immunoblotting results showed multiple bands from the leaf proteins, suggesting that Khib is widely distributed in rice seedlings. Furthermore, the blotting results displayed distinguished bands at 10-15 kD where the histones are located. However, no smear was observed at the position of histone H3 ( $15 \mathrm{kD})$.

Furthermore, we combined affinity enrichment and high-resolution LC-MS/MS methods to elucidate the 2hydroxyisobutyrylome in rice seedlings. Briefly, proteins isolated from seedling leaves were digested to peptides. Then, the peptides with Khib sites were enriched by Khib specific antibodies. Subsequent LC-MS/MS analysis was performed after affinity enrichment (Fig. 1b). MS data were validated by checking the mass errors of all the identified peptides. Most of the mass errors fell in the range of $\pm 2 \mathrm{ppm}$, with most peaks less than $0.02 \mathrm{Da}$, demonstrating high mass accuracy (Additional file 1: Fig. S1a). Additionally, the length of most of the peptides was between 7 and 20 amino acids, which is in broad agreement with the expected tryptic peptide profile, further confirming the high quality of the sample preparation (Additional file 1: Fig. S1b). Of all the 4535 peptides detected, 4129 peptides (91.0\%) with Khib were identified. In total, 4163 2-hydroxyisobutyryl lysine sites were identified on 1596 proteins in rice leaves (Additional file 2: Table S1). Furthermore, we found that 825 proteins $(51.7 \%)$ were 2-hydroxyisobutyrylated at only one site, while a very low percentage (ca. 5\%) were modified at more than eight sites (Additional file 1: Fig. S1c). Some example mass spectra of Khib-modified peptides on representative proteins are shown in Fig. S2.

\section{Identification of Khib Sites Reveals Conserved Motifs in Rice}

To investigate the amino acid sequence motifs around the Khib sites, Motif- $x$ software was used to discover conserved motifs from amino acid residues located within 10 amino acids upstream or downstream of the 2-hydroxyisobutyrylated lysine. Ten conserved motifs were detected in the rice 2-hydroxyisobutyrylome around Khib sites (Fig. 2a, b), such as EK hib, $K_{\text {hib }} K$, $\mathrm{K}_{\text {hib }} \mathrm{R}, \mathrm{DK}_{\mathrm{hib}}$ and $\mathrm{D}_{\mathrm{XX}} \mathrm{K}_{\text {hib }}\left(\mathrm{K}_{\text {hib }}\right.$ indicates the 2hydroxyisobutyrylated lysine, and $\mathrm{x}$ indicates a random amino acid residue). The motif patterns exhibit a preference for polar negatively charged amino acids, i.e., aspartate (D) and glutamate (E), flanking the modified lysine site. Similar amino acid sequence features have also been
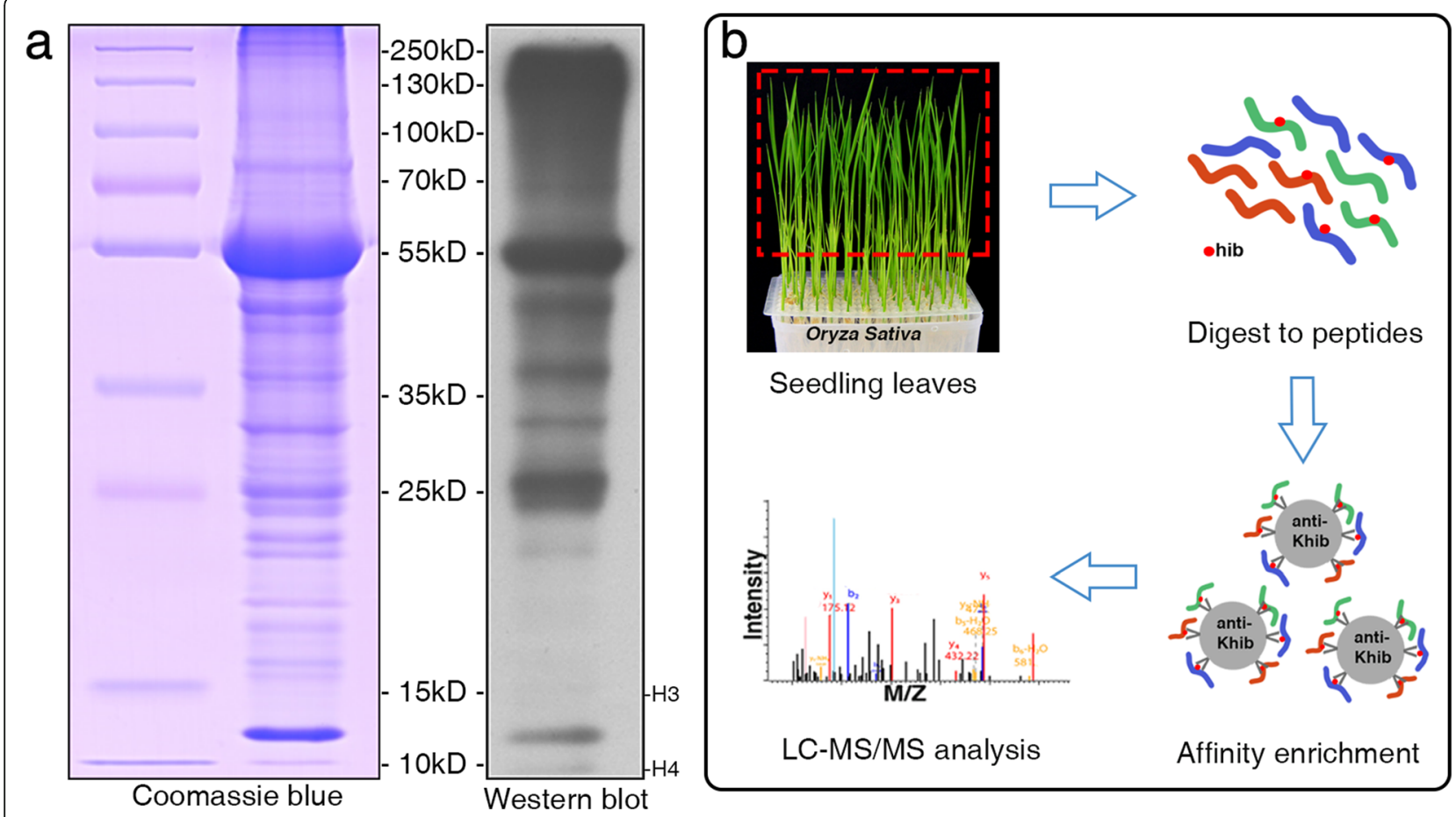

Fig. 1 Workflow for large-scale identification of Khib sites in rice. a Western blotting analysis of total proteins in rice seedling leaves. The gels for SDS-PAGE were stained with Coomassie Blue. b Workflow for identification of Khib modification in rice. Total proteins extracted from seedling leaves were digested to peptides by trypsin. Peptides bearing Khib sites were affinity enriched with a specific Khib antibody and subsequently analyzed by LC-MS/MS. 

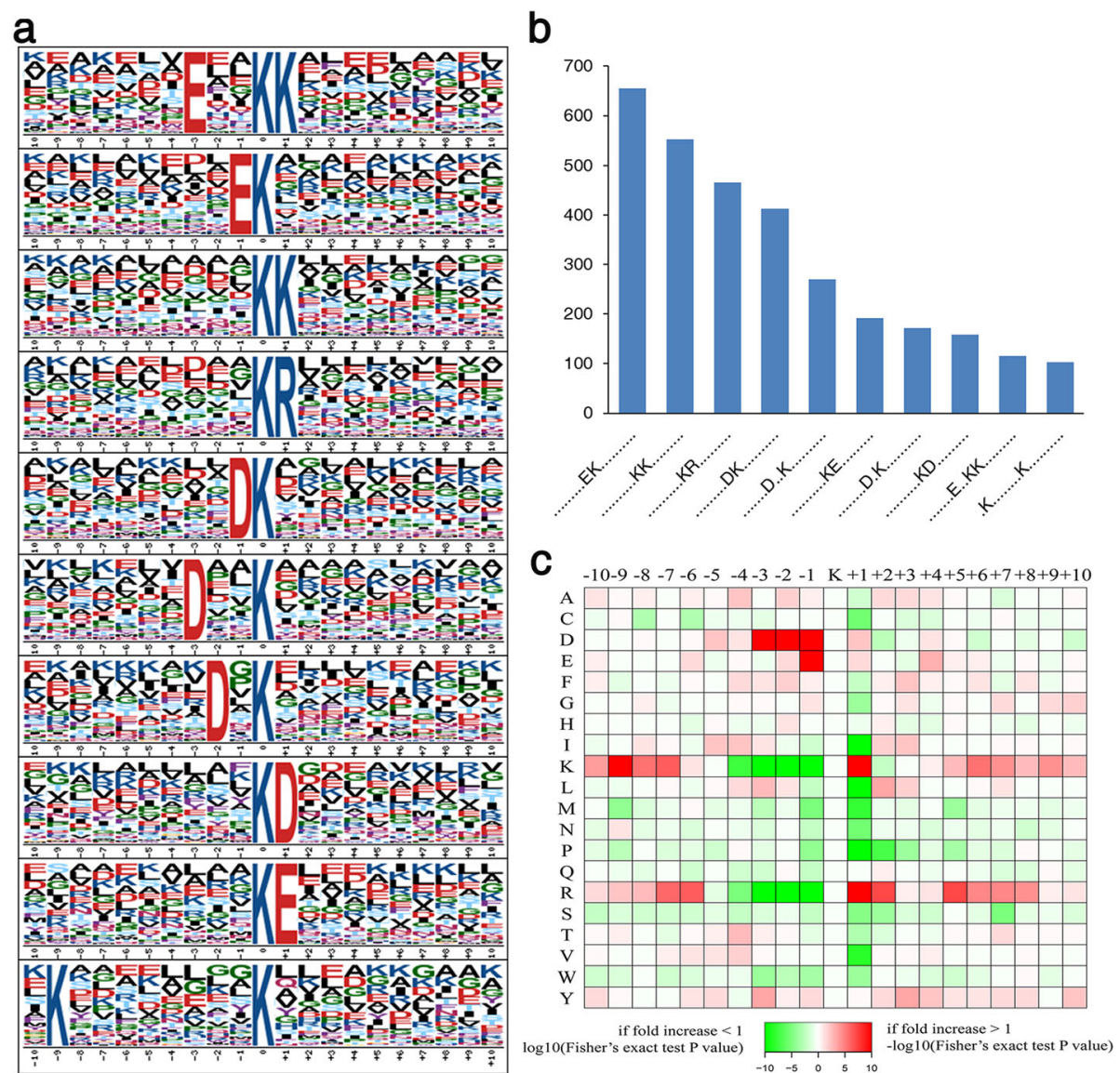

Fig. 2 Motif analysis of all identified Khib sites. a Conserved motifs identified around the Khib sites. The size of each letter reflects the frequency of that amino acid residue in that position. $\mathbf{b}$ Frequency for each motif occurring in peptides with Khib modification. $\mathbf{c}$ Heat-map of the amino acid compositions of the Khib sites, showing the enrichment (red) and depletion (green) of amino acids in each position (from -10 to +10 ) flanking the Khib sites

observed in the 2-hydroxyisobutyrylome of developing rice seeds (Meng et al. 2017). In addition, heat-map analysis of enriched and depleted amino acids showed that aspartate (D) at the $-1,-2$, and -3 positions and glutamate (E) at the -1 position are favorable for 2hydroxyisobutyrylation (Fig. 2c). Furthermore, polar positively charged amino acids, i.e., lysine (K) and arginine $(\mathrm{R})$, are overrepresented at the +1 position. However, lysine $(\mathrm{K})$ and arginine $(\mathrm{R})$ at the -1 to -4 positions are underrepresented. Thus, these results indicate that Khib is preferred at lysine residues adjacent to polar hydrophilic amino acids.

\section{Functional Annotation and Subcellular Localization Analyses of Khib Proteins}

Gene ontology (GO) analysis is typically used to ascertain gene or protein roles in eukaryotes and prokaryotes based on three independent ontologies, i.e., biological process, molecular function, and cellular component (Ashburner et al. 2000). Accordingly, to elucidate the function and distribution of Khib proteins in rice seedlings, GO classification and subcellular localization analysis were performed (Fig. 3). GO functional analysis revealed proteins mainly involved in metabolic (29\%), cellular (27\%), and singleorganism (18\%) processes and demonstrated that most Khib proteins are associated with binding (44\%) and catalytic (40\%) activity based on molecular function (Fig. 3a). Regarding the cellular component category, $38 \%$ of the Khib proteins were located in the cell, $28 \%$ in organelles, $15 \%$ in the membrane, and $14 \%$ in macromolecular complexes. The classification of Khib proteins is very similar to those that undergo other acylations in rice, such as acetylation (Ashburner et al. 2000; Meng et al. 2018; Xue et al. 2018), succinylation (He et al. 2016), and crotonylation (Liu et al. 2018), suggesting that various acylations may be involved in these molecular functions or biological processes. 

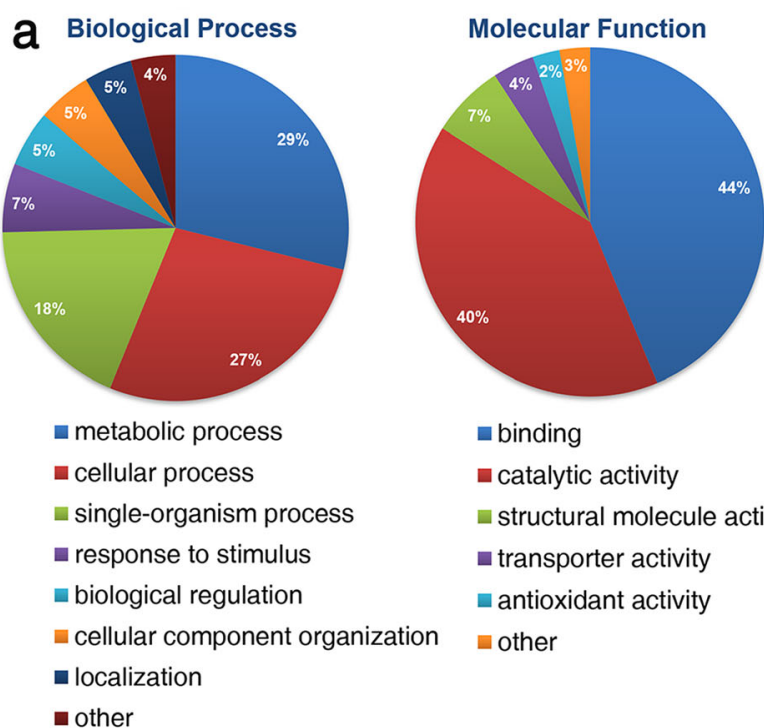

a binding

- catalytic activity

- structural molecule activity

- transporter activity

v antioxidant activity

wother

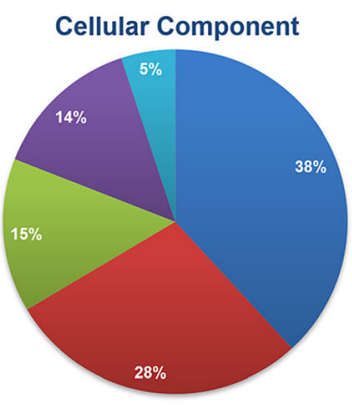

a cell

- organelle

membrane

- macromolecular complex

wother

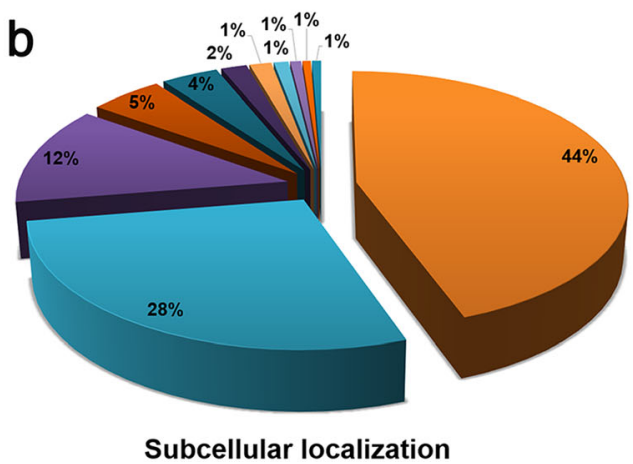
Echloroplast
cytoplasm
nucleus
mitochondria
E plasma membrane
- cytoskeleton
घextracellular
$\square$ vacuolar membrane
- peroxisome
Endoplasmic reticulum
- other

Fig. $3 \mathrm{GO}$ functional classification and subcellular localization of Khib modified proteins. a Classification of Khib proteins based on biological process, molecular function, and cellular component. b Subcellular localization of Khib proteins

The subcellular localization prediction for the modified proteins was performed using WoLF PSORT software (Fig. 3b). Most of the Khib occur in proteins located in the chloroplast (44\%), cytoplasm (28\%), and nucleus (12\%). To verify the subcellular localization analysis, we performed Western blot in the chloroplast proteins with pan2-hydroxyisobutyryllysine antibody (Additional file 1: Fig. S3). The immunoblotting results indicate that a large proportion of 2-hydroxyisobutyrylated sites were located in the chloroplast proteins. Furthermore, the functional annotation and subcellular localization results indicated that Khib may have important biological functions in diverse metabolic processes and different cellular components.

\section{Function, Pathway, and Domain Enrichment Analyses of Khib Proteins in Rice}

To investigate the metabolic processes in which the 1596 Khib proteins are involved, GO-based enrichment analysis was performed (Additional file 1: Fig. S4). The GO enrichment analysis revealed that the terms 'unfolded protein binding', 'translation factor activity', 'oxidoreductase activity', and 'chlorophyll binding' are most significantly enriched in the molecular function category. In terms of cellular components, Khib proteins are mainly enriched in the cytoplasm, plastid, chloroplast, and intracellular parts.

In terms of biological processes, the metabolic processes involving organonitrogen compounds, small molecules, carboxylic acids, organic acids, and oxoacids, as well as photosynthetic reactions, were most enriched. KEGG pathway enrichment analysis showed that proteins with Khib are involved in numerous central metabolic processes including photosynthesis, carbon metabolism, ribosomal activity, carbon fixation in photosynthetic organisms, and the citrate cycle (Fig. 4a; Additional file 1: Fig. S5). Furthermore, protein domain enrichment analysis identified 25 significantly enriched domains (Fig. 4b), including the thioredoxin-like fold, chlorophyll a/b binding protein domain, and NAD (P)-binding domain. Proteins with these domains are important in catalytic activity, photosynthesis, carbon fixation, 

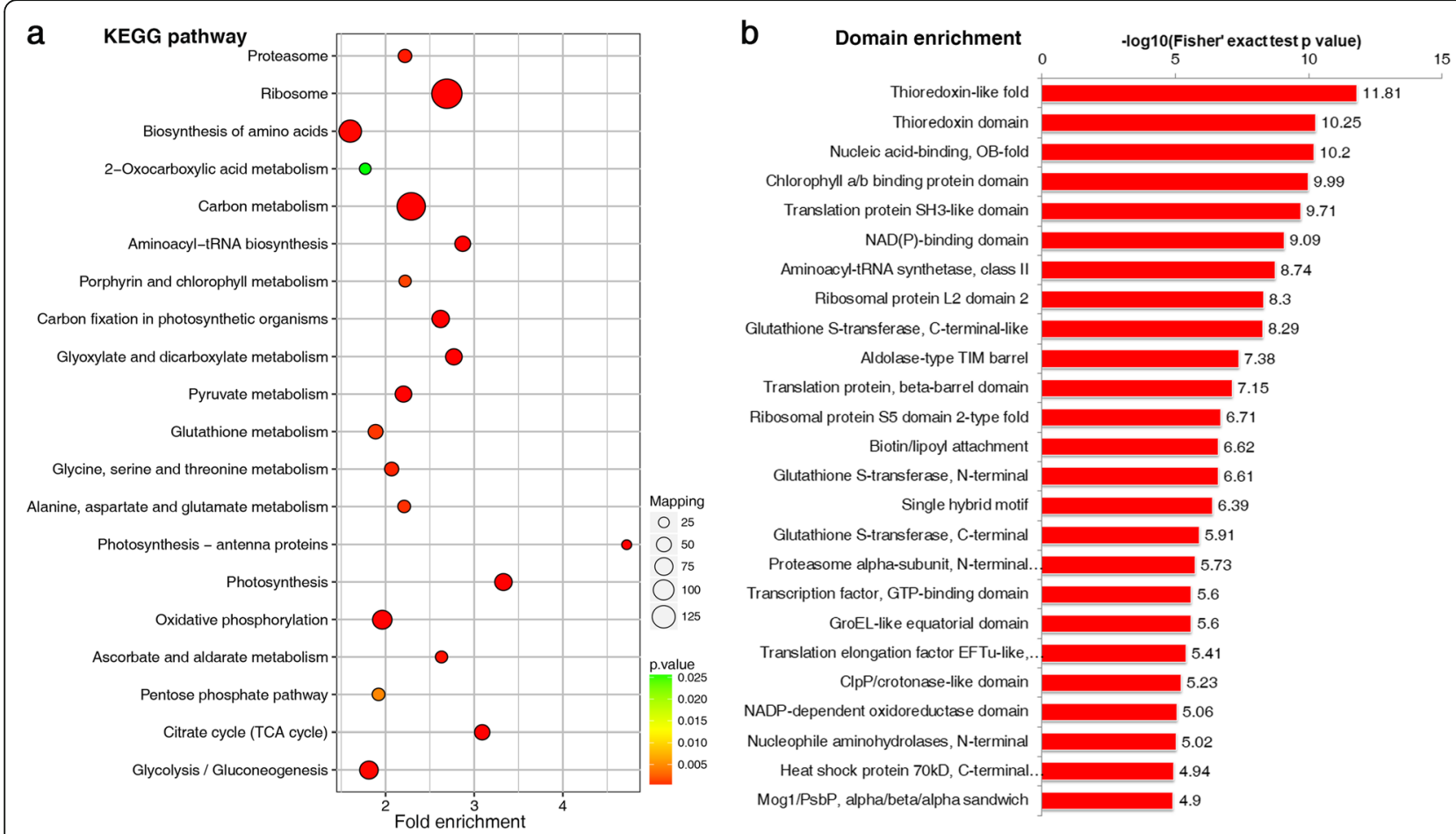

Fig. 4 KEGG pathway and Protein domain enrichment analyses of 2-hydroxyisobutyrylated proteins

and other central metabolic functions. Thus, enrichment analysis of Khib-modified proteins in rice further indicated that Khib plays pivotal roles in photosynthesis and carbon metabolism.

\section{Proteins Mostly Involved in Photosynthesis and Carbon Metabolism Are Modified by Khib}

According to the above enrichment analysis, we found that a large proportion of Khib-modified proteins are significantly associated with translation factor activity and chlorophyll binding functions, and they are enriched in photosynthesis and carbon metabolism. Thus, the vast majority of enzymes involved in the Calvin cycle and photosynthesis were mapped to further investigate the potential function of Khib in rice (Fig. 5).

A surprising number of enzymes were found to be modified by 2-hydroxyisobutyrylation, including ribulose-1,5-bisphosphate carboxylase/oxygenase (Rubisco), glyceraldehyde 3-phosphate dehydrogenase (GAPDH), phosphoglycerate kinase (PGK), and fructose1,6-bisphosphatase (FBP), as well as other key enzymes involved in carbon fixation, reduction, and regeneration. Throughout the entire photosystem in green plants, lightharvesting chlorophyll protein complex (LHC) contributes to the absorption and transfer of light energy. In rice leaves, 11 subunits of LHC (Lhca1-5 and Lhcb1-6) were found to be modified by 2-hydroxyisobutyrylation (Additional file 3: Table S2). Photosynthesis-associated proteins bearing Khib are widely distributed in cytochrome b6f complex, photosystem I and II, and ATP synthase (Fig. 5).

Overlaps of Lysine 2-Hydroxyisobutyrylation, Acetylation, and Crotonylation in Rice

With the development of high-resolution MS techniques, an increasing number of novel sites and types of acylation have been identified in rice proteins, including Kac (Xiong et al. 2016; Xue et al. 2018), Khib (Meng et al. 2017), Ksucc (He et al. 2016), Kcr (Liu et al. 2018), Kbu (Lu et al. 2018), and Kma (Mujahid et al. 2018). Based on our previous proteome profiling of Kac and Kcr in rice seedling leaves (ProteomeXchange dataset identifier PXD004870 and PXD010376; Oryza sativa variety Nipponbare) (Xue et al. 2018; Liu et al. 2018), we performed a comparative analysis with the Khib proteins identified in this study (Fig. 6).

The number of Khib sites and proteins reported in this study is higher than those in the acetylome and crotonylome identified in seedling leaves (Fig. 6a). The number of overlapped Khib proteins is 411 and 63 for Kac and $\mathrm{Kcr}$, respectively. Forty-five of the overlapped proteins for the three acylations are mainly associated with metabolic processes, oxidation reduction, and photosynthesis. In addition, 1167 (73.1\%) of the 1569 modified proteins are only 2-hydroxyisobutyrylated. Among the acylmodified sites, we identified 26 lysine sites that bear all three PTMs (Fig. 6b). The representative protein 


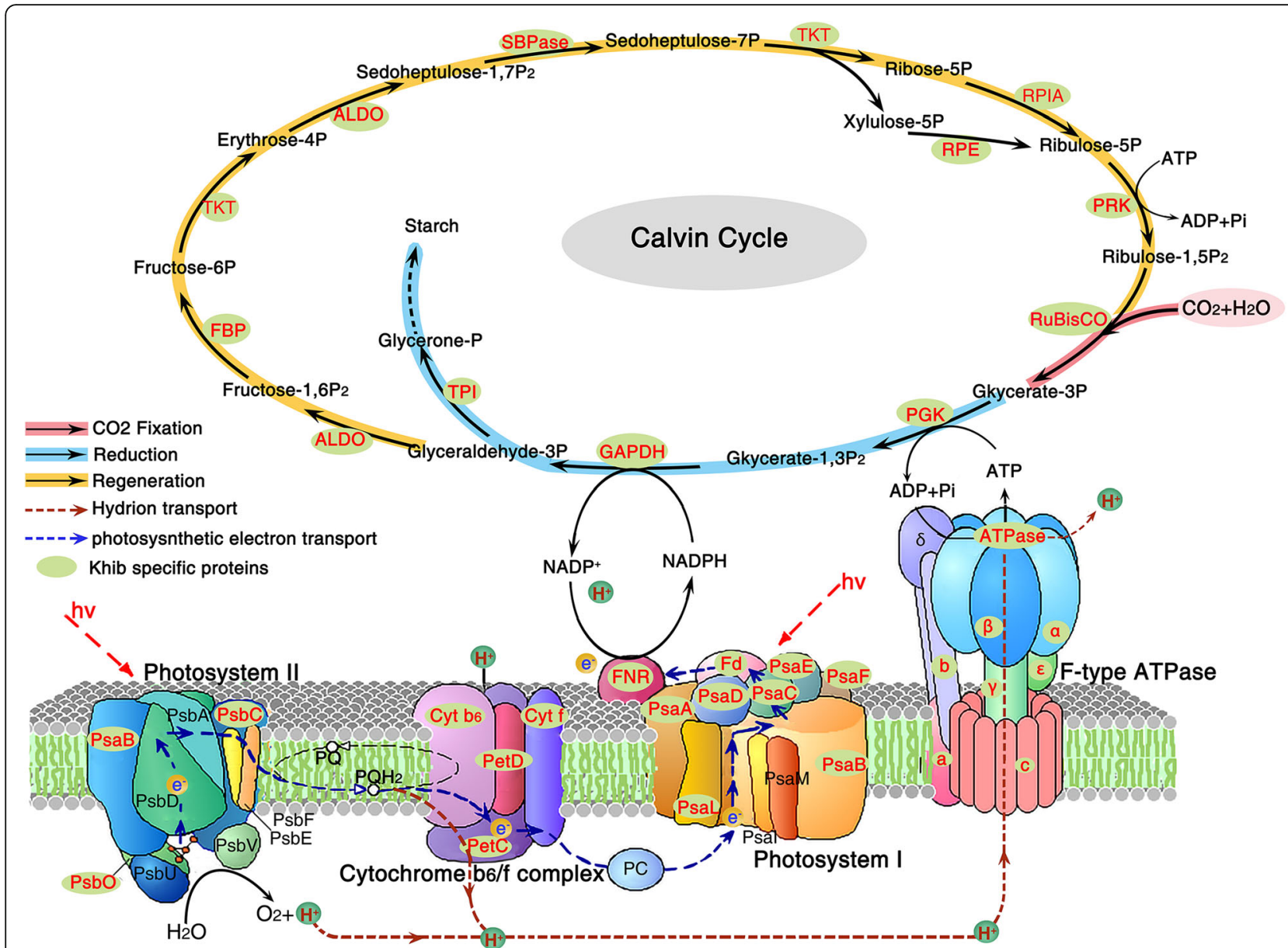

Fig. 5 Khib proteins enriched in carbon metabolism and photosynthesis pathways in rice. The 2-hydroxyisobutyrylated enzymes or proteins identified are highlighted in red

ribulose bisphosphate carboxylase/oxygenase activase (Rubisco activase, P93431), showed overlap of Khib, Kac, and Kcr (Fig. 6c; Additional file 1: Fig. S2a).

PTMs on histones are associated with diverse functions, including gene transcriptional activity, chromosome assembly, the cell cycle, and DNA damage and repair (Chen and Tian 2007; Liu et al. 2017; Sabari et al. 2017; Wang et al. 2017). Khib, as a new type of histone mark first reported in human and mouse cells, has been found to be catalyzed by histone acetyltransferases (Huang et al. 2018b; Dai et al. 2014). In this study, we identified $20 \mathrm{Khib}$ sites in core histones $(\mathrm{H} 2 \mathrm{~A} / \mathrm{H} 2 \mathrm{~B} / \mathrm{H} 4)$, among which only one site (K91) was identified in histone H4, but not in histone H3 (Fig. 6d; Table 1; Additional file 1: Fig. S2b). This is consistent with the Western blotting results (Fig. 1a). Nevertheless, seven histone Khib sites (H3K23/K56/K79/ $\mathrm{K} 122$ and $\mathrm{H} 4 \mathrm{~K} 31 / \mathrm{K} 77 / \mathrm{K} 79$ ) in humans were not identified in this study. Compared to the histone Kac and Kcr sites derived from proteome-wide acylation analyses in our previous studies (Liu et al. 2018; Xue et al. 2018), fewer Khib sites were detected in the most conserved histones $\mathrm{H} 3$ and H4. Unlike acetylation, 2-hydroxyisobutyrylation and crotonylation, as two new types of histone acylations reported in recent years, require further research to expand the proteome profiles of rice.

\section{Protein-Protein Interaction (PPI) Networks of Khib Proteins in Rice}

To further evaluate the correlation of 2-hydroxyisobutyrylated proteins in rice, we constructed a protein-protein interaction network using the STRING database and Cytoscape software (Smoot et al. 2011). We fetched all interactions that had a combined score $\geq 0.9$ (highest confidence) and identified 703 Khib proteins as nodes connected by 9834 interactions obtained from the STRING database (Version 11.0; Additional file 4: Table S3). A comprehensive PPI network of 2hydroxyisobutyrylated proteins in rice is displayed in Fig. S6. Using a clustering algorithm performed with the MCODE tool, 37 clusters of Khib proteins were retrieved from the PPI network (Additional file 4: Table S3). Based on the KEGG pathway enrichment analyses, the most abundant interaction network was identified as ribosome sub-networks, containing 
a

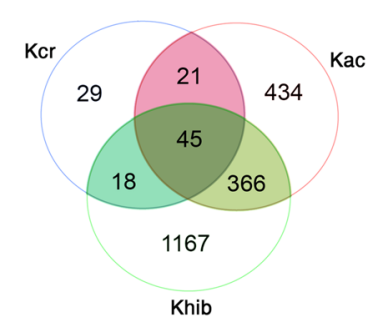

C
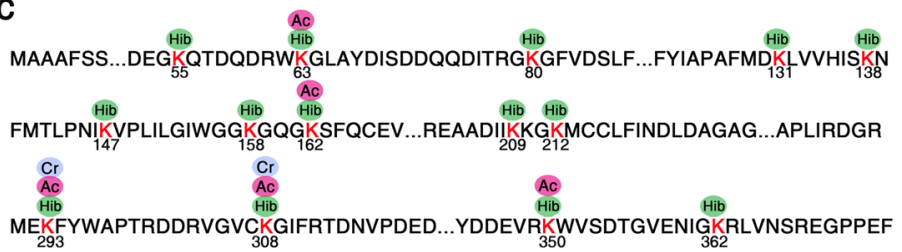

cetylation

Cr Crotonylation

Hib 2-hydroxyisobutyrylation

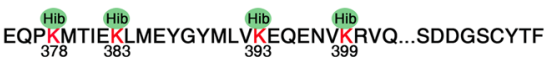

(P93431, Ribulose bisphosphate carboxylase/oxygenase activase)

d
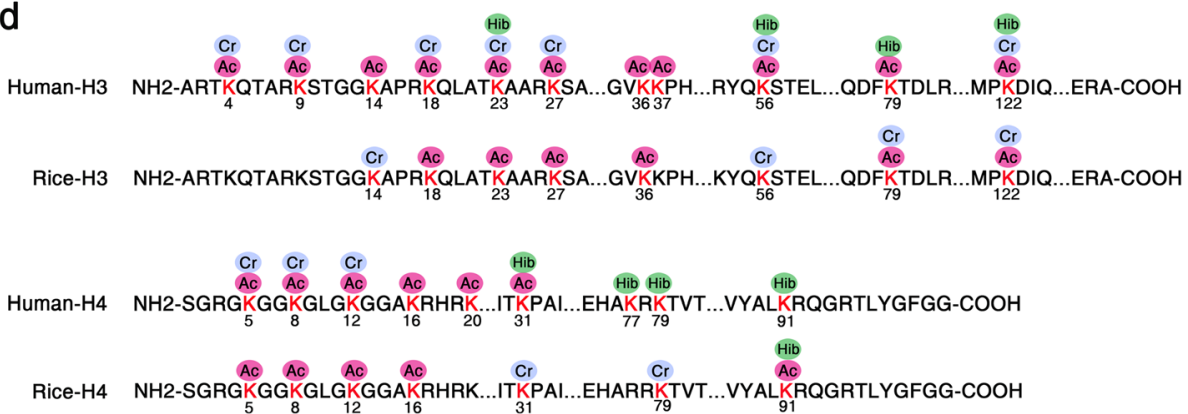

Fig. 6 Comparison of the 2-hydroxyisobutyrylome with the lysine acetylome and lysine crotonylome in rice leaves. a The overlapped number of Khib proteins among Khib, Kac, and Kcr. b The overlapped number of Khib sites among Khib, Kac, and Kcr. c Khib, Kac, and Kcr sites on the representative protein Rubisco activase (P93431). d Comparison of histone H3 and H4 with Khib, Kac, and Kcr sites between rice and human

218 ribosome-associated proteins with Khib modification (Fig. 7a). The photosynthesis and carbon metabolism subnetworks consist of 180 and 54 metabolism-associated proteins, respectively (Fig. 7b, c).

To investigate the effect of different acylations on protein function, we further analyzed the crosstalk PPI network between Kac, Kcr, and Khib modifications (Fig. 8). The crosstalk network of the 230 overlapped modified proteins (containing at least two modifications) showed that ribosome complex and biosynthesis of secondary metabolites sub-networks were highly connected.

\section{Discussion}

The catalogue of lysine acylations has been extended over the past half century, largely due to the application of high-sensitivity mass spectrometry and the development of high-specificity antibodies (Huang et al. 2014). Khib is a newly discovered PTM, first reported in human and mouse cells, and it plays a critical role in the regulation of chromatin functions (Dai et al. 2014). In the present study, we performed a global proteome identification of Khib in rice seedling leaves. A total of 4163 Khib sites on 1596 proteins were identified for expansion of the lysine 2hydroxyisobutyrylome in plants, profiling the regulatory roles of Khib in the growth and development of rice.

Compared to the previously reported 2-hydroxyisobu tyrylome in developing rice seeds (Meng et al. 2017), relatively few overlapped proteins with Khib sites and the fewer number of identified sites were observed in our dataset (Table 2). It is probably due to the completely different tissues with different types and numbers of proteins. Between the 2-hydroxyisobutyrylomes in rice seedling leaves and developing seeds, only $18.35 \%$ proteins and $8.65 \%$ lysine sites with 2-hydroxyisobutyrylation overlap. In addition, similar results have been reported for the proteome profiles of other PTMs in the same species, even in the same organs (Meng et al. 2018; Xue et al. 2018). It has been speculated that distinct sampling stages or organs lead to differences in proteome identification by the same LC-MS/MS method. Furthermore, the Khib sites and modified proteins 
Table 1 Lysine 2-hydroxyisobutyrulated sites identified in histones

\begin{tabular}{|c|c|c|c|c|}
\hline Protein accession & Position & Modified sequence & Histone description & Mass error [ppm] \\
\hline Q6ZL42 & 121 & K(0.976)AGGSAK(0.024)AAAGD & H2A.2 & -0.42403 \\
\hline Q6ZL43 & 14 & AIGAGAAK(1)K & H2A.1 & 0.15735 \\
\hline Q94E96 & 137 & TAEK(1)AAAAGK & H2A.5 & -0.281 \\
\hline Q94E96 & 11 & MDAAGAGAGGK(1)LK & H2A.5 & 0.17246 \\
\hline Q94E96 & 133 & K(0.77)TAEK(0.23)AAAAGK & H2A.5 & -0.98919 \\
\hline Q8S857 & 25 & AAADK(0.004)DK(0.996)DRK & $\mathrm{H} 2 \mathrm{~A}$ variant 2 & 2.6074 \\
\hline Q8S857 & 13 & GLLAAK(1)TTAAK & $\mathrm{H} 2 \mathrm{~A}$ variant 2 & -1.4744 \\
\hline Q84NJ4 & 14 & AIGSSAAK(1)K & H2A.3 & -0.70687 \\
\hline Q9LGH8 & 67 & SVETYK(1)IYIFK & H2B.8 & 1.4873 \\
\hline Q9LGH8 & 75 & VLK(1)QVHPDIGISSK & $\mathrm{H} 2 \mathrm{~B} .8$ & 0.13234 \\
\hline Q9LGH8 & 61 & K(1)SVETYK & $\mathrm{H} 2 \mathrm{~B} .8$ & 0.61662 \\
\hline Q9LGH8 & 108 & $\operatorname{LAGESAK(1)LAR}$ & H2B.8 & 0.77039 \\
\hline Q9LGH8 & 26 & AEK(1)APAGK & $\mathrm{H} 2 \mathrm{~B} .8$ & 0.76614 \\
\hline Q9LGH8 & 137 & LVLPGELAK(1)HAVSEGTK & $\mathrm{H} 2 \mathrm{~B} .8$ & -0.78709 \\
\hline Q9LGH8 & 145 & HAVSEGTK(1)AVTK & $\mathrm{H} 2 \mathrm{~B} .8$ & -0.72084 \\
\hline Q9LGH8 & 72 & IYIFK(1)VLK & $\mathrm{H} 2 \mathrm{~B} .8$ & -0.89643 \\
\hline Q9LGH8 & 23 & KPAEEEPAAEK(1)AEK & $\mathrm{H} 2 \mathrm{~B} .8$ & -0.20801 \\
\hline Q943L2 & 18 & KPVEEK(1)AEK & H2B.11 & -0.032173 \\
\hline Q9LGH4 & 108 & LAAEAAK(1)LAR & H2B.6 & 1.7336 \\
\hline Q6F362 & 23 & KPAEEEPAAEK(1)APAAGK & H2B.9 & 0.78845 \\
\hline Q7XUC9 & 92 & TVTAMDWYALK(1)R & $\mathrm{H} 4$ & -0.57386 \\
\hline
\end{tabular}

The number in parenthesis indicates the localization probability of Khib modification

identified in developing rice seeds or seedling leaves contribute to the expansion of the entire rice 2hydroxyisobutyrylome. Interestingly, although the 2hydroxyisobutyrylomes of developing rice seeds and seedling leaves exhibit low levels of overlapping, similar conserved motifs are observed around the Khib sites. The motif patterns exhibit a bias for polar negatively charged amino acids, i.e., $\mathrm{D}$ and $\mathrm{E}$, such as $\mathrm{EK} \mathrm{K}_{\text {hib }}$, $\mathrm{DK}$ hib, $\mathrm{K}_{\text {hib }} \mathrm{D}$,
$K_{\text {hib }} E$, and $D_{x x} K_{\text {hib }}$ (Meng et al. 2017). Like 2hydroxyisobutyrylation, a preference for amino acids D and $\mathrm{E}$ around the modified lysine residue is also observed in the crotonylomes of rice (Liu et al. 2018), tobacco (Sun et al. 2017), zebrafish (Kwon et al. 2018) and humans (Xu et al. 2017) suggesting that 2-hydroxyisobutyrylation and crotonylation are probably catalyzed by the same enzyme system. In contrast, only one common motif pattern

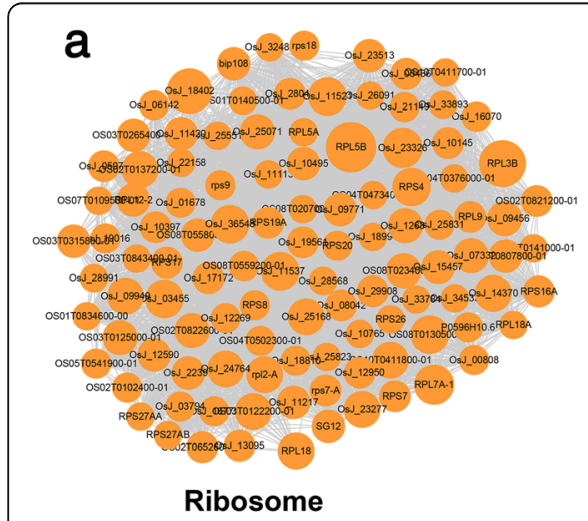

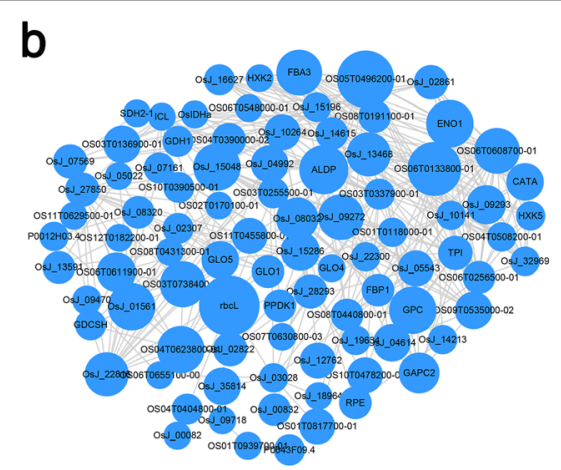

Carbon metabolism

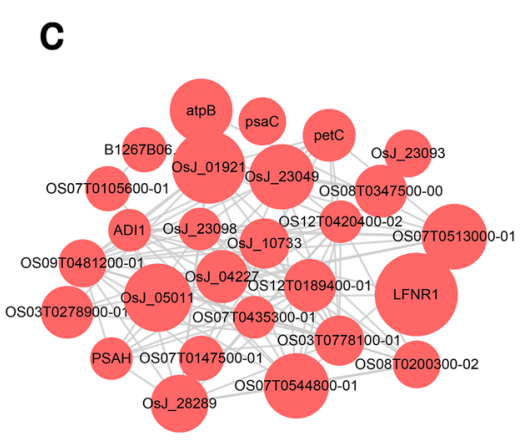

Photosynthesis

Fig. 7 The three most enriched clusters in the PPI networks of Khib proteins. The network of Khib protein interactions (listed with protein ID names) as analyzed using Cytoscape. a Ribosome. b Carbon metabolism. c Photosynthesis. The size of the dots represents the number of Khib sites in each figure 


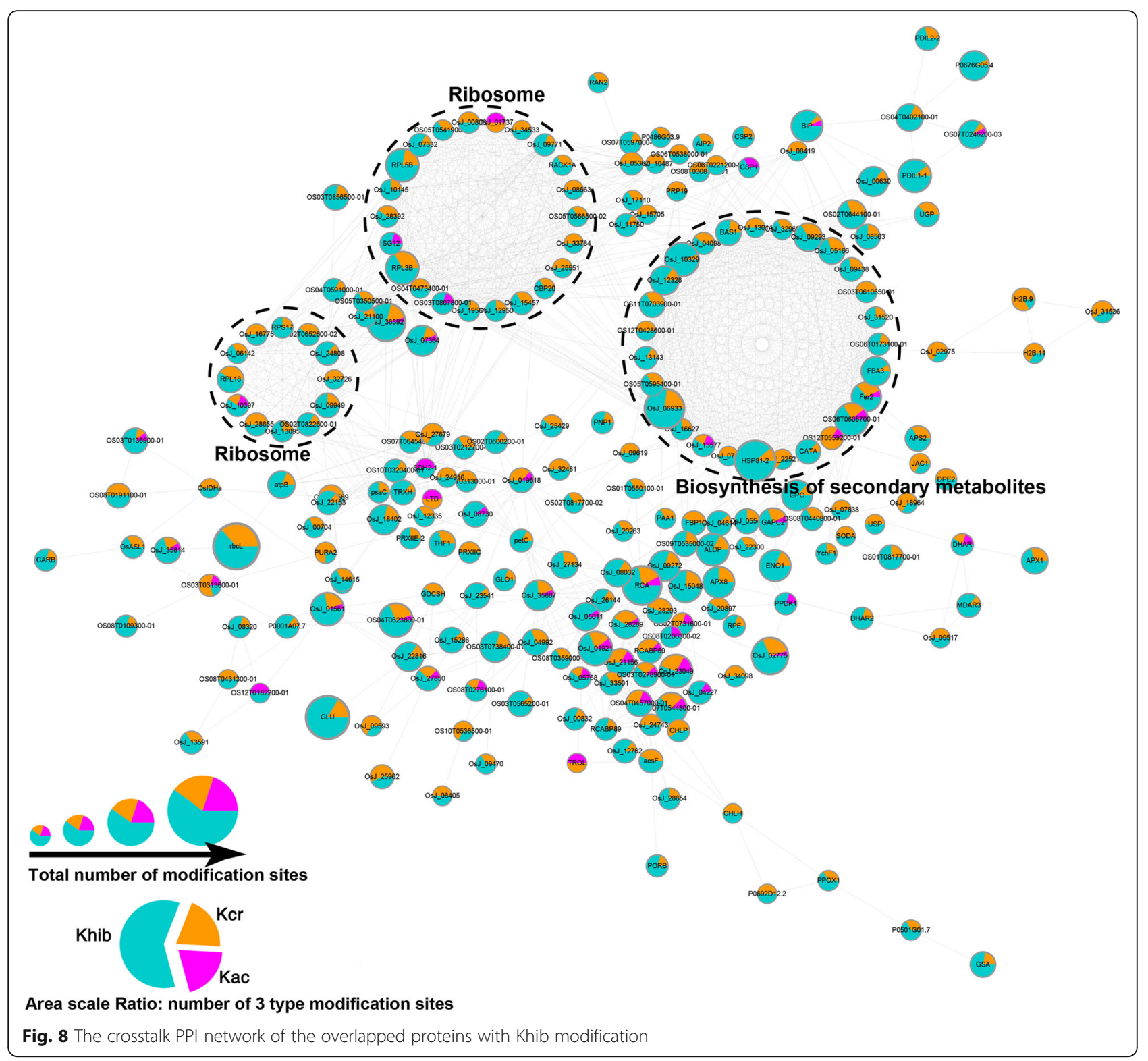

$\left(\mathrm{K}_{\mathrm{Xxxxxxxx}} \mathrm{K}_{\mathrm{PTM}}\right)$ was identified in rice 2hydroxyisobutyrylome and acetylome (Meng et al. 2018; Xue et al. 2018), which has not been reported as a Khib or Kac motif in other plants.

In our dataset, $44 \%$ of the Khib proteins in rice are located in the chloroplast, presenting high correlation with photosynthesis and carbon fixation, which is consistent with the distribution of $\mathrm{Kac}$ and $\mathrm{Kcr}$ in rice leaves (Liu et al. 2018; Xiong et al. 2016; Xue et al. 2018).
Photosynthesis is a pivotal process in green plants, converting solar energy into active chemical energy in the form of ATP and stable chemical energy in the form of sugars (Gest 2002). A large number of enzymes involved in photosynthesis and carbon metabolism were found to be 2-hydroxyisobutyrylated, including ATP synthase (subunit $\mathrm{a} / \mathrm{b} / \mathrm{c}$ and $\alpha / \beta / \gamma / \varepsilon), \mathrm{CO}_{2}$ fixation enzyme (Rubisco), reductases (GAPDH, PGK), and other photosystem complexes (photosystem I and II, cyt b6/f, and FNR).

Table 2 Overlapped Khib sites and proteins between rice seedling leaves and developing seeds

\begin{tabular}{llllll}
\hline Organs & Sampling dates & No. of Khib proteins & No. of Khib sites & Overlap (proteins/sites) & Reference \\
\hline leaves & 3-week seedlings & 1596 & 4163 & - & This study \\
developing seeds & 15 days post anthesis & 2512 & 9916 & $745 / 1219(18.35 \% / 8.65 \%)$ & (Meng et al. 2017) \\
\hline
\end{tabular}


In Arabidopsis thaliana, acetylation at the K201 and K334 sites of the key enzyme in fixing atmospheric $\mathrm{CO}_{2}$, i.e., Rubisco, negatively regulates its activity and decreases $\mathrm{CO}_{2}$ fixation efficiency (Gao et al. 2016). We also identified Khib sites on the Rubisco in rice leaves, and found Kac, Kcr, and Khib co-modified at K293 and K308 of Rubisco activase, which facilitates Rubisco remodeling (Stotz et al. 2011). Moreover, PPI network analysis of Khib proteins in this study revealed numerous sub-networks involved in the ribosome complex, biosynthesis of secondary metabolites, and metabolic pathways, including a staggering proportion of proteins located in the chloroplast (Additional file 4: Table S3). These findings suggest that Khib plays important roles in modulating carbon fixation, energy transduction, and photosynthetic efficiency in plants.

Khib was first reported as a novel and active histone mark in human and mouse cells (Dai et al. 2014). As a new type of acylation, histone Khib neutralizes the positive charge of lysine, much like Kac or Kcr does, but the distinct structure of the modifier leads to a much larger change in size and distinct genomic distribution from those of histone Kac or Kcr (Dai et al. 2014). In our study, 21 Khib sites were identified on the core histones in rice, including $\mathrm{H} 2 \mathrm{~A}$ (8 sites), $\mathrm{H} 2 \mathrm{~B}$ (12 sites), and $\mathrm{H} 4$ (only one site, K91). Unexpectedly, Khib was not detected on histone $\mathrm{H} 3$.

In the study by Dai et al., 22 histone Khib sites were detected in HeLa cells, including H3K23/K56/K79/K122 and $\mathrm{H} 4 \mathrm{~K} 31 / \mathrm{K} 77 / \mathrm{K} 79 / \mathrm{K} 91$, while 60 histone Khib sites were identified in mouse total testis cells (Dai et al. 2014). They proved that H4K8hib, like H4K8ac, is associated with transcriptional activity (Dai et al. 2014; Montellier et al. 2012). H4K91, the only overlapped Khib site on conserved histone $\mathrm{H} 4$ between our dataset and 2hydroxyisobutyrylome in humans, is in the core domain of histones rather than on the $\mathrm{NH}_{2}$-terminal tail. Previous studies have revealed that variations in H4K91 result in abnormal DNA repair and silent chromatin formation (Ma et al. 1998; Ye et al. 2005). The acetylation of the only overlapped Khib site on conserved histone $\mathrm{H} 4$ (H4K91) has been found to restrain the reassembly of histone octamers by weakening $\mathrm{H} 3 / \mathrm{H} 4-\mathrm{H} 2 \mathrm{~A} / \mathrm{H} 2 \mathrm{~B}$ binding (Ye et al. 2005). However, more information regarding the regulatory function and mechanism of histone Khib in plants and animals is required.

\section{Conclusions}

Lysine 2-hydroxyisobutyrylation is a novel protein posttranslational modification initially reported in mammals and involved in the regulation of chromatin functions. This study comprehensively profiles the lysine 2hydroxyisobutyrylome in rice leaves. Based on systematic proteomic analysis, we found that Khib occurs in proteins involved in biological functions and metabolic processes. Our results provide a better understanding of the diverse functions of lysine 2-hydroxyisobutyrylation in plants.

\section{Materials and Methods \\ Rice Seedlings and Growth Conditions}

Seedlings of Oryza sativa variety Nipponbare were grown in a growth chamber at 28 or $25^{\circ} \mathrm{C}$ in $12 \mathrm{~h}$ light/ $12 \mathrm{~h}$ dark conditions. After 3 weeks of growth, seedling leaves without stems and sheaths were excised and immediately prepared for protein extraction.

\section{Western Blot Assay}

The sample (100 mg leaf material) was ground by liquid nitrogen into powder and then transferred to a $2.0 \mathrm{~mL}$ centrifuge tube. Total proteins of the sample were prepared with extracting solution $(50 \mathrm{mM}$ Tris- $\mathrm{HCl}, 150$ $\mathrm{mM} \mathrm{NaCl}, 0.1 \% \mathrm{NP}-40,24 \%$ Urea, $1 \mathrm{mM}$ DTT, and 1 $\mathrm{mM}$ protease inhibitor). The chloroplast proteins were extracted using Chloroplast Protein Extraction Kit (BestBio. BB-3179-1) following by the manufacturer's instructions. Proteins were separated by $12 \%$ SDS-PAGE and transferred onto a PVDF membrane (Millipore). Antibody for pan-2-hydroxyisobutyryl lysine (PTM-801, PTM Biolabs) was used as the primary antibody (1:3000 dilution). Signals were detected using SuperSignal West Pico Plus chemiluminescent substrate (Thermo Scientific).

\section{Protein Extraction, Trypsin Digestion, and Affinity Enrichment}

The sample ( $1 \mathrm{~g}$ leaf material) was ground by liquid nitrogen into powder and then transferred to a $5-\mathrm{mL}$ centrifuge tube. Then, four volumes of lysis buffer $(8 \mathrm{M}$ urea, 1\% Triton-100, $10 \mathrm{mM}$ dithiothreitol, 1\% protease inhibitor cocktail, $3 \mu \mathrm{M}$ TSA and $2 \mathrm{mM}$ EDTA) was added to the tissue powder, followed by sonication three times on ice using a high intensity ultrasonic processor (Scientz). The remaining debris was removed by centrifugation at $20,000 \mathrm{~g}$ and $4{ }^{\circ} \mathrm{C}$ for $10 \mathrm{~min}$. Finally, the protein was precipitated with cold $20 \%$ TCA for $2 \mathrm{~h}$ at $20{ }^{\circ} \mathrm{C}$. After centrifugation at $12,000 \mathrm{~g}$ and $4{ }^{\circ} \mathrm{C}$ for 3 min, the supernatant was discarded. The remaining precipitate was washed with cold acetone three times. The protein was redissolved in $8 \mathrm{M}$ urea and the protein concentration was determined with a BCA kit (P0011, Beyotime Bio) according to the manufacturer's instructions.

For digestion, the protein solution was reduced with 5 $\mathrm{mM}$ dithiothreitol for $30 \mathrm{~min}$ at $56^{\circ} \mathrm{C}$ and alkylated with $11 \mathrm{mM}$ iodoacetamide for $15 \mathrm{~min}$ at room temperature in darkness. The protein sample was then diluted by adding $100 \mathrm{mM} \mathrm{NH}_{4} \mathrm{HCO}_{3}$ to provide a urea concentration of less than $2 \mathrm{M}$. Finally, trypsin was added at a 1:50 trypsin-to-protein mass ratio for the first digestion 
overnight and then at a 1:100 trypsin-to-protein mass ratio for a second 4 h-digestion.

To enrich the Khib modified peptides, tryptic peptides dissolved in IP buffer (100 mM NaCl, $1 \mathrm{mM}$ EDTA, 50 $\mathrm{mM}$ Tris- $\mathrm{HCl}, 0.5 \% \mathrm{NP}-40, \mathrm{pH} 8.0$ ) were incubated with pre-washed 2-hydroxyisobutyryl lysine antibody beads (PTM-804, PTM Biolabs) at $4{ }^{\circ} \mathrm{C}$ overnight with gentle shaking. The beads were then washed four times with IP buffer and twice with $\mathrm{H}_{2} \mathrm{O}$. The bound peptides were eluted from the beads with $0.1 \%$ trifluoroacetic acid. Finally, the eluted fractions were combined and vacuumdried. For LC-MS/MS analysis, the resulting peptides were desalted with C18 ZipTips (Millipore) according to the manufacturer's instructions.

\section{LC-MS/MS Analysis}

The tryptic peptides were dissolved in $0.1 \%$ formic acid (Solvent A), directly loaded onto a home-made reversedphase analytical column (15 cm length, $75 \mu \mathrm{m}$ i.d.). The gradient comprised an increase from $9 \%$ to $25 \%$ Solvent B ( $0.1 \%$ formic acid in $90 \%$ acetonitrile) over $38 \mathrm{~min}$, an increase from $25 \%$ to $36 \%$ over $14 \mathrm{~min}$, an increase to $80 \%$ over $4 \mathrm{~min}$, then holding at $80 \%$ for the last $4 \mathrm{~min}$, all at a constant flow rate of $300 \mathrm{~nL} / \mathrm{min}$ on an EASYnLC 1000 UPLC system.

The peptides were subjected to a nanospray ionization source followed by tandem mass spectrometry (MS/MS) on a Q Exactive ${ }^{\mathrm{mx}}$ Plus (Thermo Scientific) coupled online to the UPLC apparatus. The electrospray voltage applied was $2.0 \mathrm{kV}$. The $\mathrm{m} / \mathrm{z}$ scan range was 350 to 1800 for full scan, and intact peptides were detected in the Orbitrap at a resolution of 70,000. Peptides were then selected for MS/MS using normalized collision energy (NCE) at $28 \%$ and the fragments were detected in the Orbitrap at a resolution of 17,500. A data-dependent procedure that alternated between one MS scan followed by $20 \mathrm{MS} / \mathrm{MS}$ scans with $15.0 \mathrm{~s}$ dynamic exclusion was used. Automatic gain control (AGC) was set at 5E4.

\section{Database Search}

The resulting MS/MS data were processed using the Maxquant search engine (v1.5.2.8) (Cox and Mann 2008). Tandem mass spectra were searched against the UniProt Oryza_sativa_Japonica database (48,932 sequences) concatenated with a reverse decoy database. Trypsin/P was specified as the cleavage enzyme allowing up to four missing cleavages. The mass tolerance for precursor ions was set at $20 \mathrm{ppm}$ in the first search and at $5 \mathrm{ppm}$ in the main search, and the mass tolerance for fragment ions was set as $0.02 \mathrm{Da}$. Carbamidomethyl on Cys was specified as a fixed modification, and oxidation on Met, acetylation on the $\mathrm{N}$ terminal, and 2-hydroxyisobutyrylation on lysine were specified as variable modifications. False discovery rate (FDR) was adjusted to < $1 \%$ (Elias and Gygi 2007) and the minimum score for modified peptides was set at 40 .

\section{Motif Enrichment, GO, and Pathway Analyses}

Motif-x software (Chou and Schwartz 2011) was used for analysis of model sequences constituted by amino acids in specific positions of modifier-21-mers (10 amino acids upstream or downstream of acetylation sites) in all protein sequences. All the database protein sequences were used as background database parameters, with other parameters at default.

Proteins were classified by GO annotation (http:// www.ebi.ac.uk/GOA/) into three categories: biological process, cellular compartment, and molecular function. For each category, a two-tailed Fisher's exact test was applied to test the enrichment of the differentially expressed protein against all identified proteins. Correction for multiple hypothesis testing was carried out using standard FDR control methods. GO with a corrected $p$ value $<0.05$ is considered significant.

The Encyclopedia of Genes and Genomes (KEGG) database was used to identify enriched pathways by a two-tailed Fisher's exact test to test the enrichment of the identified modified protein against all proteins in the UniProt Oryza_sativa_Japonica database. Correction for multiple hypothesis testing was carried out using standard FDR control method, and the pathway with a corrected $\mathrm{p}$-value $<0.05$ was considered to be significantly enriched. These pathways were classified into hierarchical categories according to the KEGG website (http:// www.genome.jp/kegg/pathway.html).

\section{Khib PPI Network Analyses}

The STRING database was used to annotate the functional interactions of all the identified acetylated proteins by calculating their confidence score. Only high confidence interactions $(>0.9)$ in the STRING database (Version 11.0) were fetched for the analysis. Cytoscape software (Version 3.6.1) was used to visualize the interaction network. To identify sub-clustering groups from the protein interaction network generated by STRING, Molecular Complex Detection (MCODE) was utilized through vertex weighing by local neighborhood density and outward traversal from a local dense node (Bader and Hogue 2003).

\section{Supplementary information}

Supplementary information accompanies this paper at https://doi.org/10. 1186/s12284-020-00389-1.

Additional file 1 Fig. S1. Quality control validation of MS data and distribution of Khib peptides. Fig. S2. Representative MS/MS spectra of Khib-modified peptides in rice. Fig. S3. Western blotting analysis of chloroplast proteins in rice seedling leaves. Fig. S4. GO-based 
enrichment analysis of identified Khib proteins. Fig. S5. KEGG pathway enrichment analysis of rice Khib proteins. Fig. S6. PPI network of all identified Khib proteins.

Additional file 2 Table S1. Annotations of all identified 2hydroxyisobutyrylated proteins and the modified sites in rice seedlings. Additional file $\mathbf{3}$ Table S2. Identified Khib subunits in the chlorophyll protein complex.

Additional file 4 Table S3. Node and network information for the PPI network.

\section{Abbreviations}

Khib: Lysine 2-hydroxyisobutyrylation; LC-MS/MS: Liquid chromatographytandem mass spectrometry; PTM: Post-translational modification; KAT: Lysine acetyltransferases; KDAC: Lysine deacetylases; SDS-PAGE: Sodium dodecyl sulfate polyacrylamide gel electrophoresis; PPI: Protein-protein interaction; KEGG: Kyoto Encyclopedia of Genes and Genomes; GO: Gene Ontology; MCODE: Molecular Complex Detection; NCE: Normalized collision energy; AGC: Automatic gain control; FDR: False Discovery Rate

\section{Acknowledgements}

Not applicable.

\section{Authors' Contributions}

ZG and ZQ conceived and designed the research, $C X, X C, K L$ and $S L$ performed the experiments, PC and LY analyzed the data, CX and ZG wrote and revised the manuscript. All authors read and approved the final manuscript.

\section{Funding}

This work was supported by the National Natural Science Foundation of China [31871232, 31571266], the China Postdoctoral Science Foundation [2019 M651981], the Fund of Priority Academic Program Development of Jiangsu Higher Education Institutions (PAPD), and Suzhou Academy of Agricultural Sciences Achievement Foster Fund [KJ (18)301].

\section{Availability of Data and Materials}

The datasets used and analyzed during the current study are available from the corresponding author on reasonable request. The mass spectrometry proteomics data have been deposited to the ProteomeXchange Consortium via the PRIDE partner repository with the dataset identifier PXD018778.

\section{Ethics Approval and Consent to Participate}

Not applicable.

\section{Consent for Publication}

Not applicable.

\section{Competing Interests}

The authors declare that they have no competing interests.

\section{Author details \\ 'Jiangsu Key Laboratory of Crop Genetics and Physiology/ Key Laboratory of Plant Functional Genomics of the Ministry of Education/ Jiangsu Key Laboratory of Crop Genomics and Molecular Breeding, Agricultural College of Yangzhou University, Yangzhou 225009, China. ${ }^{2}$ Jiangsu Co-Innovation Center for Modern Production Technology of Grain Crops, Yangzhou University, Yangzhou 225009, China. ${ }^{3}$ Suzhou Academy of Agricultural Sciences, North of Wangting Town, Suzhou 215128, China.}

Received: 9 September 2019 Accepted: 11 May 2020

Published online: 05 June 2020

\section{References}

Ashburner M, Ball CA, Blake JA, Botstein D, Butler H, Cherry JM, Davis AP, Dolinski K, Dwight SS, Eppig JT, Harris MA, Hill DP, Issel-Tarver L, Kasarskis A, Lewis S, Matese JC, Richardson JE, Ringwald M, Rubin GM, Sherlock G, Consortium GO (2000) Gene ontology: tool for the unification of biology. Nat Genet 25(1):2529. https://doi.org/10.1038/75556
Ashikari M, Sakakibara H, Lin SY, Yamamoto T, Takashi T, Nishimura A, Angeles ER, Qian Q, Kitano H, Matsuoka M (2005) Cytokinin oxidase regulates rice grain production. Science 309(5735):741-745. https://doi.org/10.1126/science. 1113373

Bader GD, Hogue CW (2003) An automated method for finding molecular complexes in large protein interaction networks. BMC Bioinformatics 4:2

Chen Y, Sprung R, Tang Y, Ball H, Sangras B, Kim SC, Falck JR, Peng JM, Gu W Zhao YM (2007) Lysine propionylation and butyrylation are novel posttranslational modifications in histones. Mol Cell Proteomics 6(5):812-819. https://doi.org/10.1074/mcp. M700021-MCP200

Chen ZJ, Tian L (2007) Roles of dynamic and reversible histone acetylation in plant development and polyploidy. Biochim Biophys Acta 1769(5-6):295-307. https://doi.org/10.1016/j.bbaexp.2007.04.007

Chick JM, Kolippakkam D, Nusinow DP, Zhai B, Rad R, Huttlin EL, Gygi SP (2015) A mass-tolerant database search identifies a large proportion of unassigned spectra in shotgun proteomics as modified peptides (vol 33, pg 743, 2015). Nat Biotechnol 33(8):882-882. https://doi.org/10.1038/nbt0815-882d

Chou MF, Schwartz D (2011) Biological sequence motif discovery using motif-X. Curr Protoc bioinformatics chapter 13:unit 13 15-24. doi:https://doi.org/10. 1002/0471250953.bi1315s35

Choudhary C, Weinert BT, Nishida Y, Verdin E, Mann M (2014) The growing landscape of lysine acetylation links metabolism and cell signalling. Nat Rev Mol Cell Biol 15(8):536-550. https://doi.org/10.1038/nrm3841

Cox J, Mann M (2008) MaxQuant enables high peptide identification rates, individualized p.p.b.range mass accuracies and proteome-wide protein quantification. Nat Biotechnol 26(12):1367-1372. https://doi.org/10.1038/nbt.1511

Dai LZ, Peng C, Montellier E, Lu ZK, Chen Y, Ishii H, Debernardi A, Buchou T, Rousseaux S, Jin FL, Sabari BR, Deng ZY, Allis CD, Ren B, Khochbin S, Zhao YM (2014) Lysine 2-hydroxyisobutyrylation is a widely distributed active histone mark. Nat Chem Biol 10(5):365-U373. https://doi.org/10.1038/ Nchembio. 1497

Dong HY, Guo ZC, Feng W, Zhang T, Zhai GJ, Palusiak A, Rozalski A, Tian SS, Bai $X$, Shen LJ, Chen P, Wang Q, Fan EG, Cheng ZY, Zhang K (2018) Systematic identification of lysine 2-hydroxyisobutyrylated proteins in Proteus mirabilis. Mol Cell Proteomics 17(3):482-494. https://doi.org/10.1074/mcp. RA117. 000430

Elias JE, Gygi SP (2007) Target-decoy search strategy for increased confidence in large-scale protein identifications by mass spectrometry. Nat Methods 4(3): 207-214. https://doi.org/10.1038/nmeth1019

Gao X, Hong H, Li WC, Yang L, Huang J, Xiao YL, Chen XY, Chen GY (2016) Downregulation of Rubisco activity by non-enzymatic acetylation of RbcL. Mol Plant 9(7):1018-1027. https://doi.org/10.1016/..molp.2016.03.012

Gest H (2002) History of the word photosynthesis and evolution of its definition. Photosynth Res 73(1-3):7-10. https://doi.org/10.1023/A:1020419417954

He DL, Wang Q, Li M, Damaris RN, Yi XL, Cheng ZY, Yang PF (2016) Global proteome analyses of lysine acetylation and Succinylation reveal the widespread involvement of both modification in metabolism in the embryo of germinating Rice seed. J Proteome Res 15(3):879-890. https://doi.org/10. 1021/acs.jproteome.5b00805

Huang H, Luo ZQ, Qi SK, Huang J, Xu P, Wang XX, Gao L, Li FY, Wang J, Zhao WH, Gu W, Chen ZC, Dai LZ, Dai JB, Zhao YM (2018a) Landscape of the regulatory elements for lysine 2-hydroxyisobutyrylation pathway. Cell Res 28(1):111-125. https://doi.org/10.1038/cr.2017.149

Huang H, Sabari BR, Garcia BA, Allis CD, Zhao YM (2014) SnapShot: histone modifications. Cell 159 (2):458-+. Doi: ARTN 458.e1 181016/j.cell.2014.09.037

Huang H, Tang S, Ji M, Tang ZY, Shimada M, Liu XJ, Qi SK, Locasale JW, Roeder RG, Zhao YM, Li XL (2018b) EP300-mediated lysine 2-Hydroxyisobutyrylation regulates glycolysis. Molecular cell 70 (4):663-+. doi:https://doi.org/10.1016/j. molcel.2018.04.011

Huang J, Luo ZQ, Ying WT, Cao QC, Huang H, Dong JK, Wu QY, Zhao YM, Qian XH, Dai JB (2017) 2-Hydroxyisobutyrylation on histone H4K8 is regulated by glucose homeostasis in Saccharomyces cerevisiae. P Natl Acad Sci USA 114(33):8782-8787. https://doi.org/10.1073/pnas.1700796114

Kwon OK, Kim SJ, Lee S (2018) First profiling of lysine crotonylation of myofilament proteins and ribosomal proteins in zebrafish embryos. Sci Rep 8(1):3652. https://doi.org/10.1038/s41598-018-22069-3

Lin H, Su X, He B (2012) Protein lysine acylation and cysteine succination by intermediates of energy metabolism. ACS Chem Biol 7(6):947-960. https:// doi.org/10.1021/cb3001793

Liu S, Xue C, Fang Y, Chen G, Peng X, Zhou Y, Chen C, Liu G, Gu M, Wang K, Zhang W, Wu Y, Gong Z (2018) Global involvement of lysine Crotonylation in 
protein modification and transcription regulation in Rice. Mol Cell Proteomics 17(10):1922-1936. https://doi.org/10.1074/mcp. RA118.000640

Liu SM, Yu HJ, Liu YQ, Liu XH, Zhang Y, Bu C, Yuan S, Chen Z, Xie GJ, Li WJ, Xu B, Yang JG, He L, Jin T, Xiong YD, Sun LY, Liu XH, Han CS, Cheng ZY, Liang J, Shang YF (2017) Chromodomain protein CDYL acts as a Crotonyl-CoA Hydratase to regulate histone Crotonylation and spermatogenesis. Molecular cell 67 (5):853-+. doi:https://doi.org/10.1016/j.molcel.2017.07.011

Lu Y, Xu QT, Liu Y, Yu Y, Cheng ZY, Zhao Y, Zhou DX (2018) Dynamics and functional interplay of histone lysine butyrylation, crotonylation, and acetylation in rice under starvation and submergence. Genome biology 19. Doi: ARTN 144 https://doi.org/10.1186/s13059-018-1533-y

Ma XJ, Wu J, Altheim BA, Schultz MC, Grunstein M (1998) Deposition-related sites $\mathrm{K} 5 / \mathrm{K} 12$ in histone $\mathrm{H} 4$ are not required for nucleosome deposition in yeast. Proc Natl Acad Sci U S A 95(12):6693-6698. https://doi.org/10.1073/pnas.95. 12.6693

Martin C, Zhang Y (2005) The diverse functions of histone lysine methylation. Nat Rev Mol Cell Biol 6(11):838-849. https://doi.org/10.1038/nrm1761

Maxwell PH, Wiesener MS, Chang GW, Clifford SC, Vaux EC, Cockman ME, Wykoff CC, Pugh CW, Maher ER, Ratcliffe PJ (1999) The tumour suppressor protein VHL targets hypoxia-inducible factors for oxygen-dependent proteolysis. Nature 399(6733):271-275. https://doi.org/10.1038/20459

Meng XX, Lv YD, Mujahid H, Edelmann MJ, Zhao H, Peng XJ, Peng ZH (2018) Proteome-wide lysine acetylation identification in developing rice (Oryza sativa) seeds and protein co-modification by acetylation, succinylation, ubiquitination, and phosphorylation. Bba-Proteins Proteom 1866(3):451-463. https://doi.org/10.1016/j.bbapap.2017.12.001

Meng XX, Xing SH, Perez LM, Peng XJ, Zhao QY, Redoa ED, Wang CL, Peng ZH (2017) Proteome-wide analysis of lysine 2-hydroxyisobutyrylation in developing Rice (Oryza sativa) seeds. Scientific reports 7. Doi: ARTN 17486 https://doi.org/10.1038/s41598-017-17756-6

Montellier E, Rousseaux S, Zhao Y, Khochbin S (2012) Histone crotonylation specifically marks the haploid male germ cell gene expression program: post-meiotic male-specific gene expression. BioEssays 34(3):187-193. https:// doi.org/10.1002/bies.201100141

Mujahid H, Meng X, Xing S, Peng X, Wang C, Peng Z (2018) Malonylome analysis in developing rice (Oryza sativa) seeds suggesting that protein lysine malonylation is well-conserved and overlaps with acetylation and succinylation substantially. J Proteome 170:88-98. https://doi.org/10.1016/j.jprot.2017.08.021

Sabari BR, Tang ZY, Huang H, Yong-Gonzalez V, Molina H, Kong HE, Dai LZ, Shimada M, Cross JR, Zhao YM, Roeder RG, Allis CD (2018) Intracellular Crotonyl-CoA stimulates transcription through p300-catalyzed histone Crotonylation (vol 58, pg 203, 2015). Mol Cell 69(3):533-533. https://doi.org/ 10.1016/j.molcel.2018.01.013

Sabari BR, Zhang D, Allis CD, Zhao YM (2017) Metabolic regulation of gene expression through histone acylations. Nat Rev Mol Cell Bio 18(2):90-101. https://doi.org/10.1038/nrm.2016.140

Smoot ME, Ono K, Ruscheinski J, Wang PL, Ideker T (2011) Cytoscape 2.8: new features for data integration and network visualization. Bioinformatics 27(3): 431-432. https://doi.org/10.1093/bioinformatics/btq675

Stotz M, Mueller-Cajar O, Ciniawsky S, Wendler P, Hartl FU, Bracher A, Hayer-Hartl M (2011) Structure of green-type Rubisco activase from tobacco. Nat Struct Mol Biol 18(12):1366-U1378. https://doi.org/10.1038/nsmb.2171

Sun H, Liu X, Li F, Li W, Zhang J, Xiao Z, Shen L, Li Y, Wang F, Yang J (2017) First comprehensive proteome analysis of lysine crotonylation in seedling leaves of Nicotiana tabacum. Sci Rep 7(1):3013. https://doi.org/10.1038/s41598-01703369-6

Tan MJ, Luo H, Lee S, Jin FL, Yang JS, Montellier E, Buchou T, Cheng ZY, Rousseaux S, Rajagopal N, Lu ZK, Ye Z, Zhu Q, Wysocka J, Ye Y, Khochbin S, Ren B, Zhao YM (2011) Identification of 67 histone Marks and histone lysine Crotonylation as a new type of histone modification. Cell 146(6):1015-1027. https://doi.org/10.1016/j.cell.2011.08.008

Tan MJ, Peng C, Anderson KA, Chhoy P, Xie ZY, Dai LZ, Park J, Chen Y, Huang H, Zhang Y, Ro J, Wagner GR, Green MF, Madsen AS, Schmiesing J, Peterson BS, Xu GF, Ilkayeva OR, Muehlbauer MJ, Braulke T, Muhlhausen C, Backos DS, Olsen CA, McGuire PJ, Pletcher SD, Lombard DB, Hirschey MD, Zhao YM (2014) Lysine Glutarylation is a protein posttranslational modification regulated by SIRT5. Cell Metab 19(4):605-617. https://doi.org/10.1016/j.cmet. 2014.03.014

Tsur D, Tanner S, Zandi E, Bafna V, Pevzner PA (2005) Identification of posttranslational modifications by blind search of mass spectra. Nat Biotechnol 23(12):1562-1567. https://doi.org/10.1038/nbt1168
Wang Y, Guo YR, Liu K, Yin Z, Liu R, Xia Y, Tan L, Yang P, Lee JH, Li XJ, Hawke D, Zheng Y, Qian X, Lyu J, He J, Xing D, Tao YJ, Lu Z (2017) KAT2A coupled with the alpha-KGDH complex acts as a histone $\mathrm{H} 3$ succinyltransferase. Nature 552(7684):273-277. https://doi.org/10.1038/nature25003

Weinert BT, Scholz C, Wagner SA, lesmantavicius V, Su D, Daniel JA, Choudhary C (2013) Lysine Succinylation is a frequently occurring modification in prokaryotes and eukaryotes and extensively overlaps with acetylation. Cell Rep 4(4):842-851. https://doi.org/10.1016/j.celrep.2013.07.024

Wu Q, Ke L, Wang C, Fan PS, Wu ZW, Xu XL (2018) Global analysis of lysine 2Hydroxyisobutyrylome upon SAHA treatment and its relationship with acetylation and Crotonylation. J Proteome Res 17(9):3176-3183. https://doi. org/10.1021/acs.jproteome.8b00289

Xie X, Kang HX, Liu WD, Wang GL (2015) Comprehensive profiling of the Rice Ubiquitome reveals the significance of lysine Ubiquitination in young leaves. J Proteome Res 14(5):2017-2025. https://doi.org/10.1021/pr5009724

Xie Z, Dai J, Dai L, Tan M, Cheng Z, Wu Y, Boeke JD, Zhao Y (2012) Lysine succinylation and lysine malonylation in histones. Mol Cell Proteomics 11(5): 100-107. https://doi.org/10.1074/mcp. M111.015875

Xie Z, Zhang D, Chung D, Tang Z, Huang H, Dai L, Qi S, Li J, Colak G, Chen Y, Xia C, Peng C, Ruan H, Kirkey M, Wang D, Jensen LM, Kwon OK, Lee S, Pletcher SD, Tan M, Lombard DB, White KP, Zhao H, Li J, Roeder RG, Yang X, Zhao Y (2016) Metabolic regulation of gene expression by histone lysine beta-Hydroxybutyrylation. Mol Cell 62(2):194-206. https://doi.org/10.1016/j.molcel.2016.03.036

Xiong YH, Peng XJ, Cheng ZY, Liu WD, Wang GL (2016) A comprehensive catalog of the lysine-acetylation targets in rice (Oryza sativa) based on proteomic analyses. J Proteome 138:20-29. https://doi.org/10.1016/j.jprot.2016.01.019

Xu WZ, Wan JH, Zhan J, Li XY, He HY, Shi ZM, Zhang HQ (2017) Global profiling of crotonylation on non-histone proteins. Cell Res 27(7):946-949. https://doi. org/10.1038/cr.2017.60

Xue C, Liu S, Chen C, Zhu J, Yang X, Zhou Y, Guo R, Liu X, Gong Z (2018) Global proteome analysis links lysine acetylation to diverse functions in Oryza Sativa. Proteomics 18(1). https://doi.org/10.1002/pmic.201700036

Ye JX, Ai X, Eugeni EE, Zhang LW, Carpenter LR, Jelinek MA, Freitas MA, Parthun MR (2005) Histone H4 lysine 91 acetylation: a core domain modification associated with chromatin assembly. Molecular cell 18 (1):123-130. Doi. https://doi.org/10.1016/j.molcel.2005.02.031

Yu ZM, Ni J, Sheng W, Wang ZK, Wu YH (2017) Proteome-wide identification of lysine 2-hydroxyisobutyrylation reveals conserved and novel histone modifications in Physcomitrella patens. Scientific reports 7. Doi: ARTN 15553 https://doi.org/10.1038/s41598-017-15854-Z

Zhang ZH, Tan MJ, Xie ZY, Dai LZ, Chen Y, Zhao YM (2011) Identification of Iysine succinylation as a new post-translational modification. Nat Chem Biol 7(1): 58-63. https://doi.org/10.1038/Nchembio.495

\section{Publisher's Note}

Springer Nature remains neutral with regard to jurisdictional claims in published maps and institutional affiliations.

\section{Submit your manuscript to a SpringerOpen ${ }^{\circ}$ journal and benefit from:}

- Convenient online submission

- Rigorous peer review

- Open access: articles freely available online

- High visibility within the field

- Retaining the copyright to your article

Submit your next manuscript at $>$ springeropen.com 